\section{Current role of community- acquired methicillin-resistant Staphylococcus aureus among children with skin and soft tissue infections}

\author{
Carlos G. Teran, Sunitha Sura, \\ Tarek Mohamed, Thant Lin, \\ Marsha Medows, Donkor Cynthia, \\ Sze H. Wong
}

Department of pediatrics, Woodhull Medical and Mental Health Center, Brooklyn; New York University, School of Medicine, New York, NY, USA

\section{Abstract}

Community-acquired methicillin-resistant Staphylococcus aureus has become a wellestablished pathogen with alarming rates during the last decade. The current situation of this bacteria in pediatric infections is very limited and motivated us to conduct this study. This is a retrospective and analytical study including patients less than 18 years of age with the diagnosis of skin or soft tissue infections in 2008 and 2009 meeting the criteria of Community-acquired infection. A prevalence of $41.9 \%$ among skin and soft tissue infections was found. Inducible resistance to clindamycin was detected in $1.3 \%$ of the strains and the infection shows a seasonal predilection for summer $(\mathrm{P}=0.003) ; 57.8 \%$ of the cases required hospitalization with a mean stay of $3.3 \pm 2.5$ days. The susceptibility to clindamycin and co-trimoxazole is 88 and $97 \%$ respectively. The resistance to erythromycin has reached $92 \%$. The main diagnoses at presentation was gluteal abscess plus cellulitis (34.2\%).

The prevalence of CA-MRSA is trending up and seems to become a large burden for the health system in our community. Clindamycin is still an excellent option in the community setting since inducible clindamycin resistance is extremely low in this community. Co-trimoxazole should be kept as a reserved drug to avoid the rapid resurgence resistance in the community.

\section{Introduction}

Community-acquired methicillin-resistant Staphylococcus aureus (CA-MRSA) has become a well-established pathogen in the United States and the rest of the world. Even though a broad variety of infections are associated with this pathogen, infections localized in the skin and soft tissues are predominant among the general population.

The prevalence of CA-MRSA infections in the children population has progressively increased over the last decade, and a recently published study reported an increase from 6 to $42 \%$ in a 10 years surveillance study in the United States during the last decade. ${ }^{1}$

Similar situation is described in different latitudes of the world where the incidence and prevalence of CA-MRSA has reached alarming rates varying from 28 to $85 \%$ between during the last decade. ${ }^{2-6}$

Resistance of CA-MRSA to other types of antibiotics has developed over recent years as well. Ever since a laboratory at Johns Hopkins Hospital reported induction of clindamycin resistance in vitro in erythromycin-resistant MRSA, clinicians have been wary about using clindamycin to treat CA-MRSA infections. Fortunately, the diffusion test (D-test) can be used to reliably detect erythromycin-resistant MRSA strains with inducible clindamycin resistance. ${ }^{7}$ The prevalence of inducible macrolide-lincosamide-streptogramin B resistance (MLSBi) among $S$. aureus isolates in the community setting has also increased over the years and is currently placed between $2-14 \%$ in the United States.8,9 Consequently, ineffective antibiotic treatments and longer hospital stays create a great burden to healthcare system.

In one of the few published studies in New York City twelve years ago, CA-MRSA accounted for only $10 \%$ of infections requiring hospitalization. 10 The current situation of pediatric CA-MRSA infections in New York and Brooklyn is very limited in literature and thus motivated us to conduct this study among our children population.

\section{Materials and Methods}

This was a retrospective and analytical study conducted at Woodhull Medical Center, a community hospital currently affiliated with the New York University (NYU) and located in North Brooklyn, serving a population of 416,559 people. Institutional Review Board approval from NYU and Health and Hospitals Corporation (HHC) was obtained before the review of medical records. No informed consent was needed.

All patients less than 18 years of age with the diagnosis of skin or soft tissue infection between January 2008 and December 2009 were included initially in the review. Community-acquired (CA) infection was defined as one presenting in those patients without a history of hospitalization, surgery, or dialysis for at least one year prior to presentation; without a indwelling catheter or percutaneous medical device at the time of culture;
Correspondence: Carlos G. Teran, Woodhull Medical and Mental Health Center; New York University, New York, NY, USA.

E-mail: carlos.teran@woodhullhc.nychhc.org

Key words: MRSA, CA-MRSA, skin infections, clindamycin, children.

Acknowledgments: we appreciate the help from medical records department that facilitated the charts of the patients.

Conflict of interest: the authors report no conflicts of interest.

Contributions: CGT, case study and manuscript writing; SS, TM, TL, data collection and literature review; MM study coordination, medical records collection and manuscript writing; CD, data collection; SW, manuscript editing and literature review.

Received for publication: 13 August 2011. Accepted for publication: 19 September 2011.

This work is licensed under a Creative Commons Attribution NonCommercial 3.0 License (CC BYNC 3.0).

(C) Copyright C.G. Teran et al., 2012

Licensee PAGEPress, Italy

Pediatric Reports 2012; 4:e5

doi:10.4081/pr.2012.e5

and with infections beginning within the first 48 hours of hospitalization. Only those patients meeting the CDC criteria of community-acquired infection and positive culture of MRSA were included in the final analysis.

All cultures obtained were processed in the Microbiology Department at Kings County Hospital, Brooklyn, New York according to the National Committee for Clinical Laboratory Standards. Antimicrobial susceptibilities were determined for the following antimicrobials: clindamycin, erythromycin, moxifloxacin, gentamycin, tetracycline, rifampin and co-trimoxazole. D-Test in each strain resistant to erythromycin and sensitive to clindamycin was performed in order to detect the presence of MLSBi.

The data collected were analyzed using the JMP 6.0 statistical discovery program (SAS Institute Inc., Cary, NC, USA), and the Pearson chi-square test was used to test significance in the monthly distribution of cases.

\section{Results}

In the initial review, there were a total of 603 cases, 189 of which incision and drainage with further culture was obtained. Among the 189 cases, 126 tested positive for 
Staphylococcus aureus, and 76 of the 126 were resistant to methicillin.

Among the 76 MRSA-positive cases, 57\% were female, $43 \%$ were male; $53.9 \%$ and $46.1 \%$ presented in 2009 and 2008, respectively. When comparing the proportion of cases between months, the summer months of the year (June 13.1\%, July 18.4\%, August 15.7\%) were significantly higher than other months $(\mathrm{P}=0.003$ ) (Figure 1). 57.8\% of the cases required hospitalization with a mean stay of $3.3 \pm 2.5$ days. The majority of main diagnoses at presentation were lower extremity infections, of which gluteal abscess plus cellulitis was most common (34.2\%), followed by leg abscess (11.8\%) and knee region abscess plus cellulitis (6.5\%). Infections in the abdomen, trunk, face, and upper extremities accounted for the remaining proportion of cases.

The susceptibility pattern is visualized in Figure 2. D-Test was performed for all patients with strains resistant to erythromycin and was positive only in one case (1.3\%). Clindamycin (65.1\%), followed by cephazolin $(8.4 \%)$ and vancomycin $(5.2 \%)$ were the preferred drugs in the initial treatment for hospitalized patients. Similarly, clindamycin (54.9\%), followed by cephalexin (19.6\%) and co-trimoxazole $(9.8 \%)$ were the favorite drugs chosen as initial therapy in outpatients.

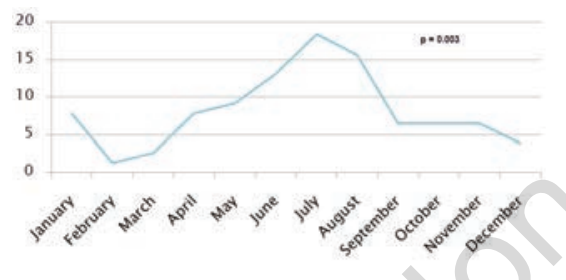

Figure 1. Seasonal variance of the occurrence of soft tissue staphylococcal infections.

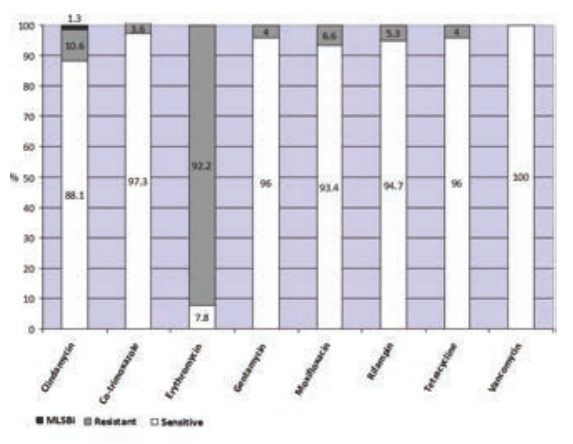

Figure 2. Sensitivity pattern for community acquired methicillin staphylococcus aureus.

\section{Discussion}

In one of the largest surveillance studies across the United States, Mera et al. recently found a five-fold increase, from 6.3 to $36.3 \%$, in the proportion of CA-MRSA isolates from children younger than fifteen between 1998 and 2007. ${ }^{1}$

Our study found a prevalence of $41.9 \%$ among cultured skin and soft tissue infections, which is alarming considering that culture was obtained only in $31 \%$ of the cases. Despite the high prevalence of their use, clindamycin and co-trimoxazole are acceptable as first-line antibiotics to treat skin and soft tissue infections in our community given CA-MRSA's susceptibility. Moxifloxacin and other less commonly used antibiotics in children such as rifampin, gentamycin, and tetracyclines possess a very low rate of resistance in our strains and should be reserved for only special situations in pediatrics. The resistance to erythromycin is alarming. Kaplan et al conducted a three-year surveillance study in Houston showing extremely high rates of resistance to erythromycin comparable with the $92.2 \%$ found in our study. ${ }^{11}$ Along the same lines, the rates of resistance to clindamycin in our study have doubled in six years in Houston and Baltimore from 6-12\%.11,12 However, despite the increased use of this antibiotic and the slight increase in its resistance, clindamycin can still be considered acceptable for clinical use. Inducible resistance to clindamycin is very low in our community (1.3\%), compared to those reported in literature in the United States (2.4-16\%) and the rest of the world (2$94 \%) .8,11,12$

Genetically distinct strains of Staphylococcus aureus circulates around the world and across the United States, conferring a variety of virulence and resistance factors. The abscense of MLSBi genotype is specially recognized among ST8:USA300 strains, the most prevalent MRSA associated with community type infections and may explain this variability in the expression of inducible resistance. ${ }^{2,13}$ One study conducted in Brooklyn determined that ST8:USA300 was the most prevalent strain in the area. 14

The presence of MLSBi and its clinical implication should be individualized in each case. Treatment failure was more frequently reported in severe systemic MRSA infections, but clindamycin may still play an important role in mild to moderate skin and soft tissue infections with similar rates of success than in the absence of MLSBi. 15

Kaplan and Szczesiul found a higher incidence of cases during warm months of the year. ${ }^{11,12}$ We found a similar seasonal variance in our study. In vitro studies have demonstrated that the optimum temperature for growth and productions of toxins for Staphylococcus aureus is around 35 to $37^{\circ} \mathrm{C}$. This can explain the tendency for peak incidence of cases in hot temperatures.

Frequent monitoring of the prevalence of antibiotic-resistances in MRSA infections is important in identifying resistance trends and guiding treatment since the presence of this frightful bacteria alone leads to unnecessary use of systemic antibiotics and costly hospitalizations, without even first trying with oral antibiotics and drainage, treatments known to be successful in the literature.

\section{Conclusions}

In conclusion, the prevalence of CA-MRSA is trending up over time and seems to become a large burden for the health system in our community as in the rest of the United States. Clindamycin is still an excellent option as firstline treatment in the community setting since inducible clindamycin resistance is extremely low in this community despite high resistance to erythromycin. Co-trimoxazole should be kept as a reserved drug for those cases not responding to other antibiotics to avoid the rapid resurgence of co-trimoxazole resistance in the community.

\section{References}

1. Mera RM, Suaya JA, et al. Increasing Role of Staphylococcus aureus and CommunityAcquired Methicillin-Resistant Staphylococcus aureus Infections in the United States: A 10-Year Trend of Replacement and Expansion. Microb Drug Resist. 2011 Mar 19. (Epub ahead of print).

2. Otter JA, French GL. Molecular epidemiology of community-associated meticillinresistant Staphylococcus aureus in Europe. Lancet Infect Dis 2010;10: 227-39.

3. Bukharie HA. Increasing threat of community-acquired methicillin-resistant Staphylococcus aureus. Am J Med Sci. 2010; 340:378-81.

4. Adam HJ, Allen VG, Currie A, et al. Community-associated methicillin-resistant Staphylococcus aureus: prevalence in skin and soft tissue infections at emergency departments in the Greater Toronto Area and associated risk factors. CJEM 2009;11:439-46.

5. Mekviwattanawong S, Srifuengfung S, Chokepaibulkit K, et al. Epidemiology of Staphylococcus aureus infections and the prevalence of infection caused by community-acquired methicillin-resistant Staphylococcus aureus in hospitalized 
patients at Siriraj Hospital. J Med Assoc Thai 2006;89Suppl5:S106-17.

6. Paganini H, Della Latta MP, Muller Opet B et al. Community-acquired methicillinresistant Staphylococcus aureus infections in children: multicenter trial. Arch Argent Pediatr 2008;106:397-403.

7. Fiebelkorn KR, Crawford SA, McElmeel ML, et al. Practical disk diffusion method for detection of inducible clindamycin resistance in Staphylococcus aureus and coagulase-negative staphylococci. J Clin Microbiol 2003;41:4740-4.

8. Patel M, Waites KB, Moser SA, et al. Prevalence of inducible clindamycin resistance among community and hospital-associated Staphylococcus aureus isolates. J Clin Microbiol 2006;44:2481-4.
9. Thomas RW, Gene H, Bradley WF. Community-Associated MethicillinResistant Staphylococcus aureus. Emerg Med Clin N Am 2008;26:431-55.

10. Rubin RJ, Harrington CA, Poon A, et al. The economic impact of Staphylococcus aureus infection in New York City hospitals. Emerg Infect Dis 1999;5:9-17.

11. Kaplan SL, Hulten KG, et al. Three-year surveillance of community-acquired Staphylococcus aureus infections in children. Clin Infect Dis 2005;40:1785-91.

12. Szczesiul JM, Shermock KM, Murtaza UI, Siberry GK. No decrease in clindamycin susceptibility despite increased use of clindamycin for pediatric community-associated methicillin-resistant Staphylococcus aureus skin infections. Pediatr
Infect Dis J 2007;26:852-4.

13. Orscheln RC, Hunstad DA, et al. Contribution of genetically restricted, methicillin-susceptible strains to the ongoing epidemic of community-acquired Staphylococcus aureus infections. Clin Infect Dis 2009;49:536-42.

14. Bratu S, Landman D, Gupta J, et al. A population-based study examining the emergence of community-associated methicillin-resistant Staphylococcus aureus USA300 in New York City. Ann Clin Microbiol Antimicrob 2006;5:29.

15. Woods, Charles R. Macrolide-Inducible Resistance to Clindamycin and the D-Test. Pediatr Infect Dis J 2009;28:1115-8. 